\title{
LMI conditions for robust adaptive control of MIMO LTI systems *
}

\author{
Dimitri Peaucelle \\ LAAS-CNRS, Université de Toulouse \\ 7, av. du colonel Roche, 31077 Toulouse, FRANCE \\ Email: peaucelle@laas.fr \\ Alexander Fradkov \\ Institute for Problems of Mechanical Engineering of RAS \\ 61 Bolshoy av. V.O., St Petersburg, 199178, RUSSIA \\ Email: alf@control.ipme.ru
}

July 3, 2007

\begin{abstract}
: Passification-based direct adaptive control is considered for polytopic uncertain linear time-invariant multi-input multi-output systems. Linear Matrix Inequality based results are provided to guarantee that the adaptive algorithm passifies the system whatever the uncertain parameters in some given set. The contributions are based on the introduction of a parallel feed-forward shunt that liberates from the strong equality constraint $P B=C^{T} G^{T}$ often used for strict passification. The shunt, combined with the introduction of slack variables, allows to produce results without assumptions on which data is uncertain in the process model. Formulas proving robust passification are formulated as Linear Matrix Inequality and can therefore be efficiently tested. A simple academic example illustrates the results. Keywords: Passivity, Robustness, Adaptive Control, LMI, output-feedback.
\end{abstract}

\section{INTRODUCTION}

Adaptive control techniques are conceived with the idea that on line modification of the control algorithm is needed in order to reject the inevitable uncertainties and disturbances. One such adaptive scheme $[6,1]$ relies on adaptive parameter estimation which gives, either directly or after some computation, the data for tuning the controller. Yet another adaptation scheme [4, 7] takes advantage of passivity properties to perform directly the tuning of the controller gains parameters with the sole output measurements. The paper is dedicated to robustness issues in this passification-based strategy (also called simplified adaptive control in [7]).

The case of state-space linear time-invariant (LTI) systems is considered ( $\dot{x}=A x+$ $B u, y=C x$ ). Passivity-based adaptive control of such systems is proved to be possible

\footnotetext{
* This work is supported by CNRS-RAS research cooperation program No. 19134 and Complex Program of the Presidium of RAS \#22 "Control processes", project 1.8 .
} 
if there exists a combination of the outputs $\hat{y}=G y$ such that the system from its inputs $u$ to the artificial output $\hat{y}$ is hyper minimum phase [5]. Equivalently it corresponds to the existence of a static output feedback $u=F y+v$ such that the closed-loop from $v$ to $\hat{y}$ is strictly passive (the open-loop system is sometimes called almost strictly passive [7] in that case). One interesting issue is that for a given matrix $G$ the problem of proving that a system is static feedback passifiable can be formulated in terms of Linear Matrix Iinequalities (LMIs) and for some cases $[2,10]$ methodologies to choose the matrix $G$ have been provided.

At this stage a question arises: why performing adaptive control if static feedback is available? The answer is that adaptive control is expected to be robust and this may be proved by the existence of a parameter-dependent passifying static feedback. In a sense this indicates, for particular situations, that the existence of an estimation/gain-tuning adaptive control implies the validity of a simple passivity-based adaptive law.

In [10], LMI-based results are derived for the existence of a parameter-dependent static feedback that passifies the system under the assumption that only the $A$ matrix of the LTI system are affected by uncertainties. This limitation may seem inevitable if considering strict passivity of systems without feed-through gains. Indeed it relies on an inequality constrain $P B=C^{T} G^{T}$ which is hard to satisfy if the $B$ and $C$ matrices are imperfectly known. Inspired by the results of [7], a parallel feedforward gain (also known as a shunt) is introduced in order to extend the results to systems where all matrices are uncertain. LMI results are produced that allow the simultaneous design of the feedback and shunt gains that both have the same polytopic structure as the uncertain system. Similarly to the results of [12] (that considers state-feedback and not output-feedback), robustness is proved using parameter-dependent storage functions thanks to the slack-variable methodology of [9].

The paper is organized as follows. First, a brief section is devoted to the description of the considered static parameter-dependent and adaptive control schemes. In particular, the adaptive control is composed of a dead-zone type function which is needed for proving closed-loop stability and which, as suggested in [1], has disturbance rejection properties. In the third section, the passification problem is formulated and LMI conditions are provided for the case of LTI systems without uncertainties. The fourth section exposes the robust LMI results and finally a numerical example is treated in the last section.

Notations: $\mathbb{R}^{m \times n}$ is the set of $m$-by- $n$ real matrices. $A^{T}$ is the transpose of the matrix $A .1$ and $\mathbf{0}$ are respectively the identity and the zero matrices of appropriate dimensions. For symmetric matrices, $A>(\geq) B$ if and only if $A-B$ is positive (semi) definite.

\section{Control strategies}

The paper considers passification of uncertain LTI systems described in state-space as $\Sigma(\Delta)$ :

$$
\dot{x}=A(\Delta) x+B(\Delta) u, \quad y=C(\Delta) x
$$

where $x \in \mathbb{R}^{n}$ is the state, $u \in \mathbb{R}^{m}$ is the control input and $y \in \mathbb{R}^{l}$ is the measurement output. $\Delta$ stands for parametric uncertainties which are supposed to belong to a given set $\boldsymbol{\Delta}$ and are assumed to be constant (or varying sufficiently slowly in comparison with the system dynamics). 
Two control strategies are adopted and compared. One is parameter-dependent static output-feedback

$$
u(t)=F(\Delta) y(t)+v(t),
$$

and supposes measurement or estimation of the uncertain parameters $\Delta$.

The second, is adaptive control defined as

$$
\begin{aligned}
& u(t)=K(t) y(t)+v(t) \\
& \dot{K}(t)=-G y(t) y^{T}(t) \Gamma-\phi_{\beta, \gamma}(K(t)) \Gamma
\end{aligned}
$$

where $\phi_{\beta, \gamma}$ is a dead-zone type function defined as $\phi_{\beta, \gamma}(K)=\mathbf{0}$ if $\operatorname{Tr}\left(K^{T} K\right) \leq \gamma$ and

$$
\phi_{\beta, \gamma}(K)=\frac{\operatorname{Tr}\left(K^{T} K\right)-\gamma}{\beta \gamma-\operatorname{Tr}\left(K^{T} K\right)} K
$$

if $\gamma \leq \operatorname{Tr}\left(K^{T} K\right)<\beta \gamma$, where $\gamma>0$ and $\beta>1$. For the scalar case, the function is as represented in Figure 1. Throughout the paper admissible initial conditions: $\operatorname{Tr}\left(K^{T}(0) K(0)\right)<\beta \gamma$ are assumed.

Figure 1: The scalar case dead-zone function for $\gamma=10$ and $\beta=2$

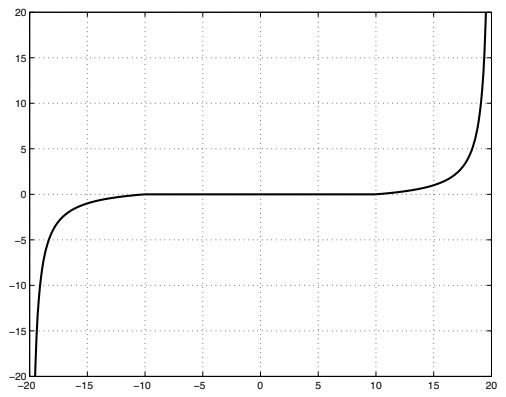

The adaptive control law is conceived such that the term $-G y(t) y^{T}(t) \Gamma$ tunes in real time the feedback gain $K(t)$ in order, hopefully, to converge towards passifying values. Conditions for that property to hold are given in the next section. The second term $-\phi_{\beta, \gamma}(K(t)) \Gamma$ is intended to guarantee bounded values for $K(t)$. As shown in $[1,10]$ such term also contributes to robustness with respect to external disturbances, in particular to noise on the measurements. Some properties of $\phi_{\beta, \gamma}$ are now given.

Lemma 1 For all $F$ satisfying $\operatorname{Tr}\left(F^{T} F\right) \leq \gamma$ and for all $K$, the following inequality holds

$$
\operatorname{Tr}\left(\phi_{\beta, \gamma}(K)(K-F)^{T}\right) \geq 0 .
$$

Proof: Take $T$ such that $F^{T} F \leq T$ with $\operatorname{Tr}(T) \leq \gamma$. A Schur complement argument gives

$$
\left[\begin{array}{cc}
T & F^{T} \\
F & \mathbf{1}
\end{array}\right] \geq \mathbf{0} .
$$

Pre and post multiply this inequality by $\left[\begin{array}{ll}1 & -K^{T}\end{array}\right]$ and by its transpose respectively to get

$$
T-F^{T} K-K^{T} F+K^{T} K \geq \mathbf{0} .
$$

Add and substract $K^{T} K$ in this expression to get

$$
(K-F)^{T} K+K^{T}(K-F) \geq K^{T} K-T
$$


which, taking the trace of the inequality, implies

$$
\begin{aligned}
& 2 \operatorname{Tr}\left((K-F)^{T} K\right)=2 \operatorname{Tr}\left(K(K-F)^{T}\right) \\
& \quad \geq \operatorname{Tr}\left(K^{T} K-T\right) \geq \operatorname{Tr}\left(K^{T} K\right)-\gamma
\end{aligned}
$$

and this allows to conclude.

Lemma 2 If $y(t)$ is bounded for all $t \geq 0$, then $\operatorname{Tr}\left(K^{T}(t) K(t)\right)<\beta \gamma$ for all $t \geq 0$.

Proof: Consider the following Lyapunov function $V(K)=\operatorname{Tr}\left(K K^{T}\right)$. Its derivatives along the trajectories writes

$$
\begin{aligned}
\dot{V}(K)= & -2 \operatorname{Tr}\left(G y y^{T} \Gamma K^{T}+\phi_{\beta, \gamma}(K) \Gamma K^{T}\right) \\
= & -y^{T}\left(\Gamma K^{T} G+G^{T} K \Gamma\right) y \\
& -2 \frac{\operatorname{Tr}\left(K^{T} K\right)-\gamma}{\beta \gamma-\operatorname{Tr}\left(K^{T} K\right)} \operatorname{Tr}\left(K^{T} \Gamma K\right) .
\end{aligned}
$$

The last term in this formula goes to infinity as $\operatorname{Tr}\left(K^{T} K\right)$ goes to $\beta \gamma$. Therefore, for bounded values of $y$ and $K$ there exists a scalar $\alpha$ such that $\dot{V}(K)<0$ for all $K$ such that $\alpha \leq \operatorname{Tr}\left(K^{T} K\right)<\beta \gamma$, i.e. the trajectories are decreasing and $\operatorname{Tr}\left(K^{T} K\right)$ cannot exceed $\beta \gamma$.

Note that if the measurements are affected by bounded noise or disturbances, then the result of lemma 2 remains valid.

\section{Closed-loop passivity}

This section considers the case of systems without uncertainties. The goal is to derive constructive LMI conditions for that case. These will allow in the next section to produce robustness results.

Let the LTI system described in state-space as $\Sigma$ :

$$
\dot{x}=A x+B u, y=C x
$$

Passification is looked for by means of either static feedback $u=F y+v$ or non-linear adaptive feedback (3). In both cases the closed-loop enters the format

$$
\dot{\eta}=f(\eta)+\hat{B} v(t), y=\hat{C} \eta
$$

where $\eta=x$ in case of static feedback and contains all the elements of $K$ in case of adaptive feedback. In this last case $f$ is a non-linear function.

For a state-space model defined by a state $\eta$, an input vector $v$ and an output vector $z$, consider the following inequality

$$
V(\eta(t)) \leq V(\eta(0))+\int_{0}^{t}\left[v(\theta)^{T} z(\theta)-\rho(\eta(\theta))\right] d \theta
$$

Based on this inequality, let the following definitions:

Definition 1 The system is said to be globally passive with respect to the signals $v, z$ if there exists a nonnegative scalar function $V(\eta)$ (storage function) and $\rho=0$ such that (6) holds for all $t \geq 0$ and all initial conditions. 
Definition 2 The system is said to be globally strictly passive with respect to the signals $v, z$ if there exists a nonnegative scalar function $V(\eta)$ and a nonnegative scalar function $\rho(\eta)$, strictly positive for all $\eta \neq 0$, such that (6) holds for all $t \geq 0$ and all initial conditions.

Definition 3 Let a partitioning of the state $\eta$ in two parts $x$ and $K$. The system is said to be globally $x$-strictly passive with respect to the signals $v, z$ if there exists a nonnegative scalar function $V(\eta)$ and a nonnegative scalar function $\rho(\eta)$, strictly positive for all $x \neq 0$, such that (6) holds for all $t \geq 0$ and all initial conditions.

All three definitions are global, i.e. they hold for all initial conditions. The adjective "global" is eluded in the remaining to alleviate the text.

All passivity conditions imply system's stability. Additionally, strict passivity implies asymptotic stability and $x$-strict passivity implies that for zero inputs $v$, the part of the state $x$ converges to zero.

Theorem 1 The closed-loop system (1) with static feedback $u=F y+v$ is strictly passive with respect to the signals $v, z=G y+D v$ if and only if there exist a symmetric positive-definite matrix $P>0$ and a positive scalar $\epsilon>0$ such that the following LMI conditions hold:

$$
\left[\begin{array}{cc}
A^{T}(F) P+P A(F) & P B \\
B^{T} P & \mathbf{0}
\end{array}\right] \leq\left[\begin{array}{cc}
-\epsilon \mathbf{1} & C^{T} G^{T} \\
G C & D+D^{T}
\end{array}\right]
$$

where $A(F)=A+B F C$.

Proof: The theorem corresponds to the positive-real lemma [3] applied to the closed-loop system $\dot{x}=A(F) x+B v, \quad z=G C x+D v$. It is also an LMI version of the SPR conditions recalled in [6].

Note that if $D=\mathbf{0}$, the inequality (7) become that of [5]

$$
A^{T}(F) P+P A(F) \leq-\epsilon \mathbf{1}, P B=C^{T} G^{T} .
$$

Any robustness tests based on this last result have the disadvantage that the $B$ and $C$ matrices need to be exactly known. Indeed, except for very special situations, the equality constraint $P B=C^{T} G^{T}$ is impossible to maintain even when assuming parameter dependent matrices $P$. Hence, the feed-through gain $D$ is an opportunity for producing robustness results with respect to uncertain systems with uncertainties affecting $B$ and C.

Theorem 2 If there exists a positive scalar $\epsilon>0$, two matrices $F, D$, and three symmetric positive-definite matrices $P>0, R>0, T>0$ such that the following LMI conditions hold

$$
\begin{gathered}
{\left[\begin{array}{cc}
R & C^{T} G^{T}-P B \\
G C-B^{T} P & \mathbf{1}
\end{array}\right] \geq \mathbf{0}} \\
{\left[\begin{array}{cc}
T & F^{T} \\
F & \mathbf{1}
\end{array}\right] \geq \mathbf{0}, \quad \operatorname{Tr}(T) \leq \gamma} \\
{\left[\begin{array}{cc}
L & P B-C^{T} G^{T} \\
B^{T} P-G C & -D-D^{T}
\end{array}\right] \leq \mathbf{0}}
\end{gathered}
$$


where

$$
\begin{aligned}
L= & A^{T} P+P A+\beta \gamma C^{T} C+\epsilon \mathbf{1} \\
& +C^{T}\left(G^{T} F+F^{T} G\right) C+R,
\end{aligned}
$$

then both control laws $u=F y+v$ and (3), $x$-strictly passify the system with respect to the signals $v, z=G y+D v$.

Proof: First, prove the result for the static feedback control. Pre and post multiply inequality (8) by [ $\left.\begin{array}{ll}1 & C^{T} F^{T}\end{array}\right]$ and by its transpose respectively to get:

$$
\begin{aligned}
& C^{T}\left(G^{T} F+F^{T} G\right) C+R \\
& \quad \geq P B F C+C^{T} F^{T} B^{T} P-C^{T} F^{T} F C .
\end{aligned}
$$

Due to this inequality, (10) implies that

$$
\left[\begin{array}{cc}
\hat{L} & P B-C^{T} G^{T} \\
B^{T} P & -D-D^{T}
\end{array}\right] \leq \mathbf{0}
$$

where

$$
\begin{array}{r}
\hat{L}=A^{T}(F) P+P A(F)+\epsilon \mathbf{1} \\
-C^{T}\left(F^{T} F-\beta \gamma \mathbf{1}\right) C .
\end{array}
$$

A Schur complement argument on (9) gives that $\operatorname{Tr}\left(F^{T} F\right) \leq \operatorname{Tr}(T) \leq \gamma$. It implies that $F^{T} F \leq \gamma \mathbf{1}$. As $\beta>1$ one gets $F^{T} F-\beta \gamma \mathbf{1} \leq \mathbf{0}$. The inequality (11) therefore implies that of Theorem 1 which ends this part of the proof.

Now, prove the result for the adaptive control law. Let any Hermitian positive definite matrix $\Gamma>0$ and let the output-feedback law (3). Consider the following storage function

$$
V(\eta)=\frac{1}{2} x^{T} P x+\frac{1}{2} \operatorname{Tr}\left((K-F) \Gamma^{-1}(K-F)^{T}\right) .
$$

Along the trajectories of (1) with the control law (3) the derivatives of $V(\eta)$ write

$$
\begin{aligned}
\dot{V}(\eta)=x^{T} P(A x+ & B K y+B v) \\
& +\operatorname{Tr}\left(\dot{K} \Gamma^{-1}(K-F)^{T}\right) .
\end{aligned}
$$

Pre and post multiply the matrix inequality (10) by $\left(\begin{array}{ll}x^{T} & v^{T}\end{array}\right)$ and its transpose respectively, to get

$$
\begin{aligned}
2 x^{T} P(A x+B v) \leq & 2 z^{T} v-\epsilon x^{T} x-x^{T} R x \\
& -2 y^{T} F^{T} G y-\beta \gamma y^{T} y .
\end{aligned}
$$

Pre and post multiply (8) by ( $\left.\begin{array}{cc}x^{T} & y^{T} K^{T}\end{array}\right)$ and its transpose respectively to get

$$
2 x^{T} P B K y-x^{T} R x \leq 2 y^{T} K^{T} G y+y^{T} K^{T} K y .
$$

Combining these two last inequalities, the derivatives of $V(\eta)$ satisfy the inequality

$$
\begin{array}{r}
\dot{V}(\eta) \leq z^{T} v-\frac{\epsilon}{2} x^{T} x+y^{T}(K-F)^{T} G y \\
+y^{T}\left(K^{T} K-\beta \gamma \mathbf{1}\right) y \\
+\operatorname{Tr}\left(\dot{K} \Gamma^{-1}(K-F)^{T}\right) .
\end{array}
$$


Note the following result based on the fact that $\operatorname{Tr}\left(M_{1} M_{2}\right)=\operatorname{Tr}\left(M_{2} M_{1}\right)$ :

$$
\begin{array}{r}
y^{T}(K-F)^{T} G y=\operatorname{Tr}\left(y^{T}(K-F)^{T} G y\right) \\
=\operatorname{Tr}\left(G y y^{T}(K-F)^{T}\right) .
\end{array}
$$

Therefore, replacing $\dot{K}$ by its value, one obtains

$$
\begin{array}{r}
\dot{V}(\eta) \leq z^{T} v-\frac{\epsilon}{2} x^{T} x+y^{T}\left(K^{T} K-\beta \gamma \mathbf{1}\right) y \\
-\operatorname{Tr}\left(\phi_{\beta, \gamma}(K)(K-F)^{T}\right) .
\end{array}
$$

The last quantity is negative due to Lemma 1 . Moreover, Lemma 2 guarantees that $\operatorname{Tr}\left(K^{T} K\right) \leq \beta \gamma$ and hence $K^{T} K-\beta \gamma \mathbf{1} \leq \mathbf{0}$. Taking the integral over time of $\dot{V}(\eta)$ on finally gets (6) with $\rho(\eta)=\frac{1}{2} \epsilon x^{T} x$ which concludes the proof.

\section{Guaranteed Robustness}

This section is aimed at demonstrating that the LMI conditions derived upper allow to produce robustness results. The uncertain system $\Sigma(\Delta)$ of (1) is assumed of polytopic type. The dependency with respect to the uncertain parameters $\Delta=\left(\zeta_{1}, \ldots, \zeta_{N}\right)$ is affine such that

$$
\begin{gathered}
{\left[\begin{array}{cc}
A(\Delta) & B(\Delta) \\
C(\Delta) & \mathbf{0}
\end{array}\right]=\sum_{i=1}^{N} \zeta_{i}\left[\begin{array}{cc}
A_{i} & B_{i} \\
C_{i} & \mathbf{0}
\end{array}\right]} \\
\zeta_{i} \geq 0, \sum_{i=1}^{N} \zeta_{i}=1
\end{gathered}
$$

where the $A_{i}, B_{i}$ and $C_{i}$ matrices define the vertices of the polytope.

Theorem 3 If there exists two matrices $H_{1}$ and $H_{2}$ and $6 N$ elements $P_{i}>\mathbf{0}, \epsilon_{i}>0$, $F_{i}, D_{i}, R_{i}>\mathbf{0}, T_{i}>\mathbf{0}$ such that the following LMI conditions hold for all $i=1 \ldots N$

$$
\begin{gathered}
{\left[\begin{array}{ccc}
R_{i} & C_{i}^{T} G^{T} & P_{i} \\
G C_{i} & \mathbf{1} & \mathbf{0} \\
P_{i} & \mathbf{0} & \mathbf{0}
\end{array}\right]+H_{1}\left[\begin{array}{c}
\mathbf{0} \\
B_{i}^{T} \\
\mathbf{1}
\end{array}\right]^{T}+\left[\begin{array}{c}
\mathbf{0} \\
B_{i}^{T} \\
\mathbf{1}
\end{array}\right] H_{1}^{T} \geq \mathbf{0}} \\
{\left[\begin{array}{cc}
T_{i} & F_{i}^{T} \\
F_{i} & \mathbf{1}
\end{array}\right] \geq \mathbf{0}, \operatorname{Tr}\left(T_{i}\right) \leq \gamma} \\
\tilde{L}_{i}+H_{2}\left[\begin{array}{cc}
A_{i}^{T} & C_{i}^{T} \\
B_{i}^{T} & \mathbf{0} \\
-\mathbf{1} & \mathbf{0} \\
\mathbf{0} & \mathbf{- 1}
\end{array}\right]+\left[\begin{array}{cc}
A_{i}^{T} & C_{i}^{T} \\
B_{i}^{T} & \mathbf{0} \\
\mathbf{- 1} & \mathbf{0} \\
\mathbf{0} & \mathbf{- 1}
\end{array}\right] H_{2}^{T} \leq \mathbf{0}
\end{gathered}
$$

where $\tilde{L}_{i}=$

$$
\left[\begin{array}{cccc}
R_{i}+\epsilon_{i} & -C_{i}^{T} G^{T} & P_{i} & \mathbf{0} \\
-G C_{i} & -D_{i}-D_{i}^{T} & \mathbf{0} & \mathbf{0} \\
P_{i} & \mathbf{0} & \mathbf{0} & \mathbf{0} \\
\mathbf{0} & \mathbf{0} & \mathbf{0} & \beta \gamma \mathbf{1}+F_{i}^{T} G+G^{T} F_{i}
\end{array}\right]
$$

then both control laws $u=F(\Delta) y+v$ and (3), $x$-strictly passify the system with respect to the signals $v, z=G y+D(\Delta) v$ where $F(\Delta)=\sum_{i=1}^{N} \zeta_{i} F_{i}$ and $D(\Delta)=$ $\sum_{i=1}^{N} \zeta_{i} D_{i}$. 
Proof: Define $P(\Delta)=\sum_{i=1}^{N} \zeta_{i} P_{i}, \epsilon(\Delta)=\sum_{i=1}^{N} \zeta_{i} \epsilon_{i}, R(\Delta)=\sum_{i=1}^{N} \zeta_{i} R_{i}$ and $T(\Delta)=\sum_{i=1}^{N} \zeta_{i} T_{i}$. Note that the LMIs (13), (14) and (15) are all linear with respect to the vertex matrices (with indices $i$ ). Therefore if the LMIs hold for all vertices then they also hold for all the elements of their convex hull. Denote (13- $\Delta),(14-\Delta)$ and $(14-\Delta)$ the obtained parameter-dependent inequalities. Note that $(14-\Delta)$ is exactly the parameter-dependent version of (9). Pre and post multiply (13- $\Delta$ ) by

$$
\left[\begin{array}{ccc}
\mathbf{1} & \mathbf{0} & \mathbf{0} \\
\mathbf{0} & \mathbf{1} & B^{T}(\Delta)
\end{array}\right]
$$

and its transpose respectively. The operation eliminates the slack variable $H_{1}$ and the result happens to be exactly the parameter-dependent version of (8). Pre and post multiply $(15-\Delta)$ by

$$
\left[\begin{array}{cccc}
\mathbf{1} & \mathbf{0} & A^{T}(\Delta) & C^{T}(\Delta) \\
\mathbf{0} & \mathbf{1} & B^{T}(\Delta) & \mathbf{0}
\end{array}\right]
$$

and its transpose respectively. The operation eliminates the slack variable $H_{2}$ and the result happens to be exactly the parameter-dependent version of (10). Hence, it is shown that the conditions of Theorem 2 hold for all admissible uncertainties, which concludes the proof.

Some remarks about the result:

- To apply the control law $u=F(\Delta) y+v$ it is needed to measure the uncertain parameters or at least to have an estimate of these. This is not the case for the adaptive control law (3).

- The control laws make the closed-loop passive with respect to a parameterdependent output signal $z=G y+D(\Delta) v$. If, for some backstepping design procedure for example, the output signal $z$ is needed to be reconstructed exactly whatever the uncertain parameters, then the LMI conditions may be solved for a unique matrix $D$ by constraining $D_{i}=D$ identical for all vertices.

- Note that if the LMI conditions hold for two different sets of vertices then the adaptive control law (3) proves to passify the system for the union of the two convex hulls. A gain scheduling strategy of the type $u=F(\Delta) y+v$ would in this case be even more complex to perform because it would need to switch form one polytopic description of $F(\Delta)$ to another depending on the convex hull in which the parameters lie.

\section{Example}

Let the following data

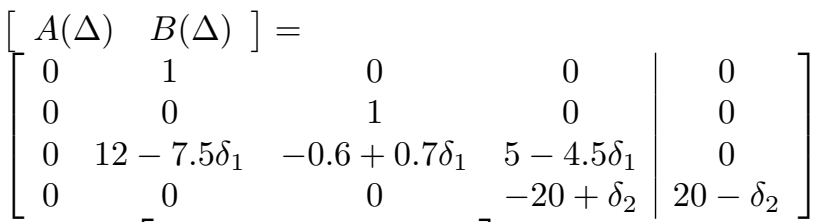

$$
\begin{aligned}
& C(\Delta)=\left[\begin{array}{cccc}
1 & 2 & 0 & 0 \\
0 & 1 & 2 & 0 \\
0 & 0 & 0 & 1+0.1 \delta_{2}
\end{array}\right], G=\left[\begin{array}{lll}
400 & 300 & 200
\end{array}\right]
\end{aligned}
$$


where $\delta_{2} \in\left[\begin{array}{ll}0 & 2.5\end{array}\right]$ and $\delta_{1} \geq-1$. The objective is to prove closed-loop stability for the largest interval on $\delta_{1}$. The tests are made considering $\gamma=10$ and $\beta=2$. The adopted procedure is to check whether the LMIs of Theorem 3 are feasible for various intervals. The results are given in Table 1. For some intervals the LMIs are found infeasible although the following tests prove the existence of some solution to the control problem. This illustrates that the LMI conditions are only sufficient and that the conservatism is reduced as the uncertainty set is reduced. Globally, taking the union of all feasible intervals, allows to conclude that the adaptive control law (3) is valid for the entire uncertainty set $\left(\delta_{1}, \delta_{2}\right) \in\left[\begin{array}{ll}-1 & 0.722\end{array}\right] \times\left[\begin{array}{ll}0 & 2.5\end{array}\right]$.

\begin{tabular}{|c|c|}
\hline$\delta_{1}$ & result \\
\hline $\left.\begin{array}{ll}-1 & 0.7\end{array}\right]$ & feasible \\
\hline$-1 \quad 0.72]$ & infeasible \\
\hline $\left.\begin{array}{ll}0.7 & 0.72\end{array}\right]$ & feasible \\
\hline $\left.\begin{array}{ll}0.7 & 0.73\end{array}\right]$ & infeasible \\
\hline $0.72 \quad 0.722]$ & feasible \\
\hline 0.723 & infeasible \\
\hline
\end{tabular}

To illustrate the impact of the uncertainties on the $B$ and $C$ matrices, the same test is performed while assuming $\delta_{2}=0$. The results are given in Table 2 .

$\underline{\text { Table 2: LMI tests for } \delta_{2}=0}$

\begin{tabular}{cc}
\hline$\delta_{1}$ & result \\
\hline$\left[\begin{array}{ll}-1 & 0.82\end{array}\right]$ & feasible \\
{$\left[\begin{array}{ll}-1 & 0.83\end{array}\right]$} & infeasible \\
{$\left[\begin{array}{cc}0.8 & 0.836\end{array}\right]$} & feasible \\
0.837 & infeasible \\
\hline
\end{tabular}

The numerical burden of the LMI problems solved in this example is characterized by 240 scalar decision variables and 121 rows in the LMI constraints. The latest version of SeDuMi [11] (SeDuMi 1.1 available at http://sedumi.mcmaster.ca/) is used along with the parser YALMIP [8]. The computation time of each individual LMI problem is less than 0.4 seconds (Linux PC computer with i686 processor and 2GB memory). No numerical problems are encountered.

To illustrate the efficiency of the adaptive control law, some simulations are performed. Three parameter configurations are tested and in each case the measurement output $y(t)$ and the control gains $K(t)$ are plotted. Figures 2, 3 and 4 correspond respectively to the configurations $\left(\delta_{1}=-1, \delta_{2}=2.5\right),\left(\delta_{1}=0.722, \delta_{2}=0\right)$ and $\left(\delta_{1}=0.722, \delta_{2}=2.5\right)$. In all experiments initial conditions are zero and a disturbance noise is applied on the measurements. This noise is a random step modified every 20 seconds.

For the values $\left(\delta_{1}, \delta_{2}\right)$ in the uncertainty set the closed-loop is stable as expected, but the dynamics are not all identical. The convergence rate is increased and bigger oscillations are observed when taking large values of the uncertainties.

Conservatism of the LMI conditions can be observed when performing simulations for values outside the proved admissible domain. For example, the choice $\left(\delta_{1}=\right.$ $\left.0.85, \delta_{2}=0\right)$ gives a stable (highly oscillating) closed-loop indicating that the infeasi- 
Figure 2: $y(t)$ and $K(t)$ for $\left(\delta_{1}=-1, \delta_{2}=2.5\right)$
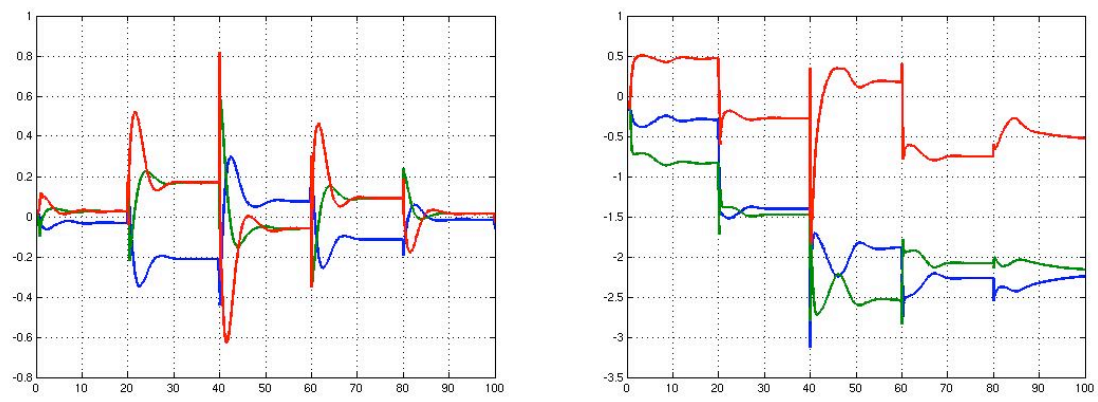

Figure 3: $y(t)$ and $K(t)$ for $\left(\delta_{1}=0.722, \delta_{2}=0\right)$
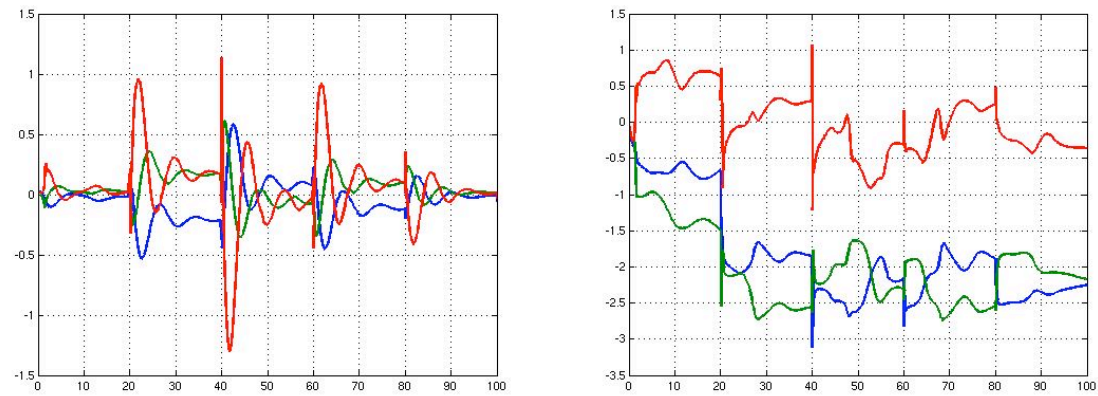

bility given for $\delta_{1}=0.837$ in Table 2 does not imply instability. For $\left(\delta_{1}=0.86, \delta_{2}=\right.$ 0 ) the closed-loop is unstable (diverges).

\section{Conclusions}

New LMI based results are provided for proving robust passification via direct passificationbased adaptive control in the case of uncertain polytopic LTI systems. The efficiency of the result is illustrated on an example. Nevertheless, many issues remain to explore. One of which is to consider other uncertain representations such as LFT modeling as it is done in [10]. Other open questions are the performances that may be acheived with such control strategies in terms of perturbation rejection or convergence rate. But the main issue to be considered is the design of the $G$ matrix. To illustrate the impact of the choice of a $G$ matrix, the same LMI constraints as above are solved for $G=\left[\begin{array}{lll}40 & 30 & 20\end{array}\right]$. In that case the maximal uncertainty set is $\left(\delta_{1}, \delta_{2}\right) \in\left[\begin{array}{ll}-1 & 0.031\end{array}\right] \times\left[\begin{array}{ll}0 & 2.5\end{array}\right]$, which clearly indicates that the choice of $G$ is a critical question. As it is done in [10] for the case without feed-through gains, we will investigate in the future some numerical procedure to derive "good" choices of $G$ matrices. The results will probably be more complex than pure LMI problems. 
Figure 4: $y(t)$ and $K(t)$ for $\left(\delta_{1}=0.722, \delta_{2}=2.5\right)$
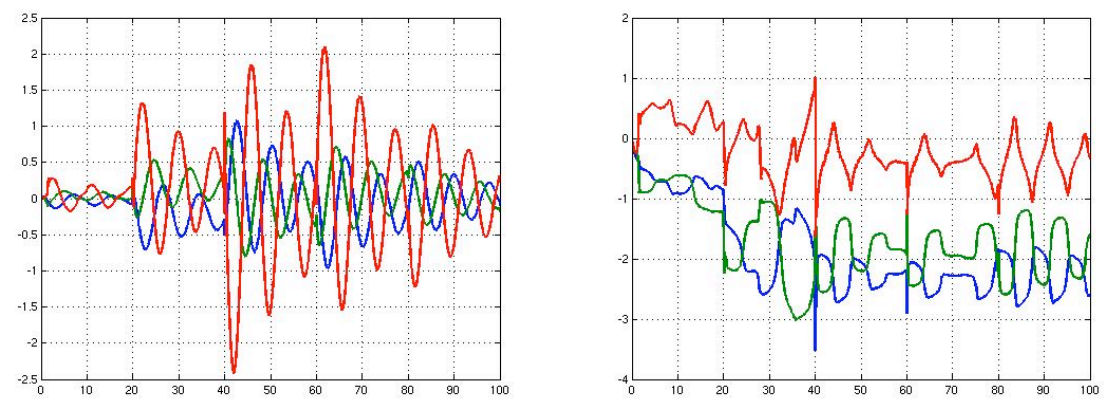

\section{References}

[1] K.J. Åström and B. Wittenmark. Adaptive Control. Addison-Wesley, 1989.

[2] I. Barkana, M.C.M. Teixeira, and L. Hsu. Mitigation of symmetry condition in positive realness for adaptive control. Automatica, 42(9):1611-1616, September 2006.

[3] S. Boyd, L. El Ghaoui, E. Feron, and V. Balakrishnan. Linear Matrix Inequalities in System and Control Theory. SIAM Studies in Applied Mathematics, Philadelphia, 1994.

[4] A.L. Fradkov. Adaptive stabilization of an linear dynamic plant. Autom. Remote Contr., 35(12):1960-1966, 1974.

[5] A.L. Fradkov. Passification of non-square linear systems and feedback Yakubovich-Kalman-Popov lemma. European J. of Control, 6:573-582, 2003.

[6] P. Ioannou and J. Sun. Robust Adaptive Control. Prentice Hall, Inc, 1996.

[7] H. Kaufman, I. Bar-Kana, and K. Sobel. Direct adaptive control algorithms. Springer, New York, 1994.

[8] J. Löfberg. YALMIP : A Toolbox for Modeling and Optimization in MATLAB, 2004.

[9] D. Peaucelle, D. Arzelier, O. Bachelier, and J. Bernussou. A new robust Dstability condition for real convex polytopic uncertainty. Systems \& Control Letters, 40(1):21-30, May 2000.

[10] D. Peaucelle, A.L. Fradkov, and B.R. Andrievsky. Passification-based adaptive control : Robustness issues. In IFAC Symposium on Robust Control Design, Toulouse, July 2006. Paper in an invited session.

[11] J.F. Sturm. Using SeDuMi 1.02, a MATLAB toolbox for optimization over symmetric cones. Optimization Methods and Software, 11-12:625-653, 1999. URL: http: / / sedumi.mcmaster.ca/.

[12] I. Yaesh and U. Shaked. Simplified adaptive control via improved robust positive real conditions. Systems \& Control Letters, 55:1033-1040, 2006. 